\title{
Low plasma concentrations of arginine and asymmetric dimethylarginine in premature infants with necrotizing enterocolitis
}

\author{
Milan C. Richir ${ }^{1}$, Michiel P. C. Siroen ${ }^{1}$, Ruurd M. van Elburg ${ }^{2}$, Willem P. F. Fetter ${ }^{2}$, Freeke Quik $^{2}$, \\ Robert J. Nijveldt ${ }^{1}$, Hugo A. Heij ${ }^{1}$, Bert J. Smit ${ }^{3} \dagger$, Tom Teerlink ${ }^{4}$ and Paul A. M. van Leeuwen ${ }^{1}$. \\ ${ }^{1}$ Department of Surgery, VU University Medical Center, Amsterdam, The Netherlands \\ ${ }^{2}$ Department of Pediatrics, VU University Medical Center, Amsterdam, The Netherlands \\ ${ }^{3}$ Department of Neonatology, Emma Children's Hospital Academic Medical Center, Amsterdam, The Netherlands \\ ${ }^{4}$ Department of Clinical Chemistry, VU University Medical Center, Amsterdam, The Netherlands
}

(Received 13 June 2006 - Revised 8 December 2006 - Accepted 8 December 2006)

\begin{abstract}
Several studies have described reduced plasma concentrations of arginine, the substrate for nitric oxide synthase (NOS) in infants with necrotizing enterocolitis (NEC). No information on the plasma concentrations of the endogenous NOS inhibitor asymmetric dimethylarginine (ADMA) in patients with NEC is currently available. We investigated whether plasma concentrations of arginine, ADMA, and their ratio differ between premature infants with and without NEC, and between survivors and non-survivors within the NEC group. In a prospective case-control study, arginine and ADMA concentrations were measured in ten premature infants with NEC (median gestational age $193 \mathrm{~d}$, birth weight $968 \mathrm{~g}$ ), and ten matched control infants (median gestational age $201 \mathrm{~d}$, birth weight $1102 \mathrm{~g}$ ), who were admitted to the Neonatal Intensive Care Unit. In the premature infants with NEC, median arginine and ADMA concentrations $(\mu \mathrm{mol} / \mathrm{l})$, and the arginine:ADMA ratio were lower compared to the infants without NEC: $21.4 v .55 .9, P=0.001 ; 0.59 v .0 .85, P=0.009$ and $36.6 v .72 \cdot 3, P=0.023$ respectively. In the NEC group, median arginine $(\mu \mathrm{mol} / 1)$ and the arginine:ADMA ratio were lower in non-surviving infants than in surviving infants: $14.7 v .33 \cdot 8, P=0.01$ and $32 \cdot 0 v$. $47.5, P=0.038$ respectively. In premature infants with NEC not only the NOS substrate arginine, but also the endogenous NOS inhibitor ADMA and the arginine:ADMA ratio were lower than in infants without NEC. In addition, low arginine and arginine:ADMA were associated with mortality in infants with NEC. Overall, these data suggest that a diminished nitric oxide production may be involved in the pathophysiology of NEC, but this needs further investigation.
\end{abstract}

Necrotizing enterocolitis: Asymmetric dimethylarginine: Dimethylarginine: Arginine: Premature infant

Necrotizing enterocolitis (NEC), is the most common gastrointestinal emergency in the premature infant (Neu, 1996). Although NEC is a multifactorial disease, prematurity is a main risk factor. Mucosal injury resulting from ischemia, bacterial colonization and early enteral feeding are also recognized as potentially important contributors to the pathogenesis of NEC (Kliegman et al. 1993; Neu, 2005).

It has been shown that in premature infants at the time of diagnosis of NEC, arginine plasma levels are decreased (Zamora et al. 1997; Becker et al. 2000). Arginine is one of the most versatile amino acids in mammalian cells, which is crucial for ammonia detoxification and serves as the precursor of NO (Wu \& Morris, 1998; Wu et al. 2004). NO plays an important role in several pathophysiological aspects of critical illness such as infection, organ injury, but also in inducing gut smooth muscle relaxation, the regulation of mucosal blood flow and maintaining mucosal integrity and the intestinal barrier function (Stark \& Szurszewski, 1992; Alican \& Kubes, 1996).
In an experimental piglet model, it was shown that arginine could have therapeutic potential in NEC by attenuating intestinal injury, whereas administration of the NO synthase (NOS) inhibitor L-NAME caused haemorrhagic congestion of the gut wall (Di Lorenzo et al. 1995). Previously, we showed in a rat model that low arginine plasma levels in combination with a low dose of endotoxin compromised blood flow through the small intestine (Nijveldt et al. 2000). Interestingly, Amin et al. (2002) showed that arginine supplementation in premature infants, reduced the incidence of all stages of NEC.

Recent insights into NO metabolism have shown an important role of endogenously produced inhibitors of NOS, in particular asymmetric dimethylarginine (ADMA; Vallance \& Leiper, 2004). ADMA as well as symmetric dimethylarginine (SDMA), are synthesized when arginine residues in proteins are methylated by the action of protein arginine methyltransferases. ADMA is an endogenous inhibitor of all isoforms of NOS, while SDMA is not.

\footnotetext{
Abbreviations: NEC, necrotizing enterocolitis; ADMA, asymmetric dimethylarginine; SDMA, symmetric dimethylarginine; NOS, nitric oxide synthase; CAT, cationic amino acid transporters; DDAH, dimethylarginine dimethylaminohydrolase.

$\dagger$ Present address: Department of Neonatology, Sophia Children's Hospital Erasmus University Medical Center, Rotterdam, The Netherlands.

* Corresponding author: Professor Paul A. M. van Leeuwen, fax +31 204443620 , email pam.vleeuwen@vumc.nl
} 
Arginine is transported into endothelial cells by the cationic amino acid transporters (CAT) of system $\mathrm{y}^{+}$, where it serves as a substrate for NO synthesis (Closs et al. 1997). Thus, the arginine:ADMA ratio is an important determinant of NO production by NOS (Bode-Boger et al. 1996; Boger et al. 1998).

Elevated plasma levels of ADMA have been found in diseases related to endothelial dysfunction including peripheral arterial disease, hypertension, hyperlipidemia, diabetes mellitus and hyerhomocysteinemia (Siroen et al. 2006). Interestingly, ADMA have been shown to independently predict intima media thickness of the carotid artery in individuals without clinical manifestations of arteriosclerosis (Miyazaki et al. 1999; Nanayakkara et al. 2005). Furthermore, it has been shown that ADMA is an independent risk factor for CHD and in critically ill patients an independent risk factor for intensive-care-unit mortality (Nijveldt et al. 2003a; Schulze et al. 2006).

We hypothesize that in addition to low arginine levels, premature infants with NEC have an increased plasma concentration of ADMA. Increased ADMA levels are associated with reduced NO synthesis and may consequently lead to a decreased mucosal blood flow and barrier function of the gut.

Therefore, the primary aim of this study was to measure the arginine, ADMA, and SDMA concentrations in premature infants with NEC and in control infants without NEC. The secondary aim was to study the relation between arginine, ADMA, and SDMA concentrations and mortality in premature infants with NEC.

\section{Methods}

Study design

We performed a prospective case-control study with a 1:1 ratio of case to control subjects. The hospital ethics committee approved the study and all infants were included after written informed consent of their parents.

\section{Patients}

All infants with a gestational age $<34$ weeks (assessed by last menstrual period of the mother and/or ultrasound before 20 weeks gestation) and a birth weight $\leq 2000 \mathrm{~g}$ admitted to the level III neonatal intensive care unit of the VU University Medical Center or the Academic Medical Center, Amsterdam, who developed NEC, were eligible for participation in the study. Only patients with NEC $\geq$ grade II diagnosed between 08.00 hours and 20.00 hours were included in the study. Exclusion criteria were major congenital or chromosomal anomalies.

NEC was diagnosed and classified according to the criteria of Bell et al. (1978). NEC grade I was characterized by nonspecific systemic signs and abdominal signs including increased gastric residuals or abdominal distension. NEC grade II encompasses the signs of grade I, absent bowel sounds or abdominal tenderness. The abdominal radiograph shows intestinal dilation, ileus and pneumatosis intestinalis. NEC grade III is characterized by bowel perforation visualized as pneumoperitoneum on the abdominal radiograph. An independent paediatric radiologist made the diagnosis of pneumatosis intestinalis.
According to the protocol guidelines for parenteral and enteral nutrition, all infants received either breast milk with breast milk fortifier (intake about $57 \mathrm{mg}$ arginine $/ 100 \mathrm{ml}$; Dupont, 2003), and/or a formula feeding (Nenatal Start ${ }^{\circledR}$, Nutricia Nederland BV, Zoetermeer, The Netherlands, containing $71 \mathrm{mg}$ arginine $/ 100 \mathrm{ml}$ ) and/or total parenteral nutrition (a hospital pharmacy based all-in-one mixture, containing $102 \mathrm{mg}$ arginine $/ 100 \mathrm{ml}$ ). After the diagnosis of NEC, a peripheral venous blood sample $(0.3 \mathrm{ml})$ was collected and the infants were treated according to the standard protocol for NEC; nothing by mouth, broad-spectrum antibiotics and parenteral feeding. If this treatment failed or in the case of intestinal perforation, surgery was performed.

\section{Control infants}

For each premature infant with NEC, one premature infant without NEC was selected. The selection was based on the characteristics of the NEC patient including gestational age, birth weight, head circumference, Apgar score at $5 \mathrm{~min}$ and postnatal age at the time of the NEC diagnosis. After inclusion, a peripheral venous blood sample $(0 \cdot 3 \mathrm{ml})$ was collected.

\section{Plasma analysis of arginine, symmetric and asymmetric dimethylarginine}

The blood samples taken from the premature infants were immediately placed on ice and centrifuged at $3000 \mathrm{rpm}$ for $10 \mathrm{~min}$ at $4^{\circ} \mathrm{C}$. Plasma was immediately put in liquid $\mathrm{N}_{2}$, and stored at $-80^{\circ} \mathrm{C}$ before analysis.

The concentration of ADMA, arginine and SDMA were determined simultaneously by HPLC as described previously (Teerlink et al. 2002). In brief, solid-phase extraction on polymeric cation-exchange columns was performed using monomethylarginine as the internal standard. After derivatization with $o$-phthaldialdehyde reagent containing 3-mercaptopropionic acid, analytes were separated by isocratic reversed-phase HPLC with fluorescence detection. Intra- and inter-assay CV were better than 1.2 and $3.0 \%$, respectively. The arginine:ADMA ratio was calculated.

\section{Statistical analysis}

Due to non-normal distribution of the amino acids, non-parametric tests were used, and data are presented as median and range. The Mann-Whitney $U$ test was used to investigate differences in plasma amino acid concentrations between the premature infants with NEC and the control group, and between surviving and non-surviving NEC patients. A value of $P<0.05$ was considered statistically significant. Statistical analysis was performed using SPSS 11.0 for Windows (SPSS Inc., Chicago, IL, USA).

\section{Results}

Between September 2001 and October 2003, a total of thirtythree premature infants with NEC were eligible to enter the study. Of these thirty-three infants, twenty-three were excluded from the study (thirteen infants with NEC grade I and ten infants who were diagnosed and treated at night or 
during the weekend). The ten premature infants with NEC $\geq$ grade II were included in the study. Six premature infants with NEC were treated by surgery, while four infants were treated with antibiotics and parenteral nutrition only. The control group consisted of ten case-controlled premature infants without NEC. Patient and nutrition characteristics are presented in Table 1 . The patient characteristics were not different between the NEC and control group. Breast milk feeding, parenteral nutrition energy intake and the intake of arginine, carbohydrate, protein and fat were not different between the two groups. Plasma concentrations of arginine, ADMA, SDMA and the arginine:ADMA ratio are shown in Table 2. The arginine concentration in premature infants with NEC was lower $(P=0.001)$ compared to the infants without NEC. The ADMA concentration in infants with NEC was also lower $(P=0.009)$ compared to the control group. Plasma SDMA was not different between the two groups. The arginine:ADMA ratio was lower $(P=0.023)$ in the NEC group.

Of the ten NEC patients, four patients died because of circulatory and respiratory insufficiency. In the non-surviving NEC infants, arginine and arginine:ADMA concentrations were lower $(P=0 \cdot 01, P=0.038$ respectively) than in the surviving infants (Table 3 ).

The plasma concentrations of the premature infants without NEC and the surviving NEC patients are shown in Table 4. The plasma concentration of arginine, ADMA and SDMA were lower in the surviving NEC patients $(P=0.022$, $P=0.011, P=0.022$ respectively). The arginine:ADMA ratio was not different between the two groups.

\section{Discussion}

Our study shows that premature infants with NEC have significantly lower plasma concentrations of arginine than premature infants without NEC. The lower concentration of arginine in NEC patients is in line with the results of other studies (Zamora et al. 1997; Becker et al. 2000). In addition, ADMA and the arginine:ADMA ratio are also significantly lower in premature infants with NEC. Furthermore, we found in non-surviving NEC patients a significantly lower arginine concentration and arginine:ADMA ratio than in surviving NEC patients. However, compared to the control patients, the surviving NEC patients had still significantly lower arginine and ADMA concentrations, but the arginine:ADMA ratio was not significantly different. These results indicate that NEC patients have less substrate available for NO synthesis, which is important to sustain blood flow in the gut of these premature infants. Moreover, these results suggest that a decreased availability of substrate for NO synthesis is associated with an increased risk of mortality.

In adult mammals, approximately $60 \%$ of net arginine synthesis occurs in the kidney, whereas in neonates, arginine is synthesized in the enterocytes of the small intestine ( $\mathrm{Wu} \&$ Morris, 1998). Wu \& Knabe (1995) studied the arginine synthesis in the enterocytes of fetal piglets and concluded that there was little synthesis of arginine in the enterocytes of preterm piglets. This near absence of intestinal arginine synthesis in preterm piglets is in sharp contrast to the high rate of intestinal arginine synthesis in term piglets ( $\mathrm{Wu}, 1997)$. In the enterocytes of preterm piglets, there is low activity of the $\Delta 1$-pyrroline-5-carboxylate synthase enzyme, which may limit the conversion of glutamine into citrulline $(\mathrm{Wu} \&$ Knabe, 1995). Furthermore, there is negligible expression of argininosuccinate synthase and argininosuccinate lyase. Both enzymes are essential for the conversion of citrulline into arginine. Notwithstanding that these studies are performed in animal models, decreased expression of argininosuccinate synthase and argininosuccinate lyase and the incapability of the fetal kidney to synthesize arginine may account for the decreased level of arginine in premature infants.

Arginine is a physiological precursor for the synthesis of NO which plays an important role in regulating vascular tone, the development of gastrointestinal circulation, and in preserving the integrity of the gastrointestinal mucosal barrier (Stark \& Szurszewski, 1992; Alican \& Kubes, 1996; Wu \& Morris, 1998; Wu et al. 2004). Conversely, the NOS inhibitor L-NNA preferentially reduces the gastrointestinal blood flow as shown in fetal sheep (Fan et al. 1996). In a rat model, the NOS inhibitor

Table 1. Nutritional and patient characteristics of premature infants with necrotizing enterocolitis (NEC) and without NEC (control)

(Median values and ranges)

\begin{tabular}{|c|c|c|c|c|}
\hline & \multicolumn{2}{|c|}{$\operatorname{NEC}(n 10)$} & \multicolumn{2}{|c|}{ Control (n 10) } \\
\hline & Median & Range & Median & Range \\
\hline Male: Female & \multicolumn{2}{|c|}{$5: 5$} & \multicolumn{2}{|c|}{$6: 4$} \\
\hline Gestational age (weeks) & $27 \cdot 6$ & $25.6-31.9$ & $28 \cdot 7$ & $26 \cdot 3-33 \cdot 3$ \\
\hline Birth weight (g) & 968 & $700-1822$ & 1102 & $690-1710$ \\
\hline Head circumference $(\mathrm{cm})$ & 28 & $22-35$ & 28 & $23-30$ \\
\hline Apgar score at $5 \mathrm{~min}$ & 9 & $3-10$ & 8 & $4-9$ \\
\hline Postnatal age $(d)$ & 16 & $7-38$ & 14 & $7-30$ \\
\hline Breast milk feeding $(n)$ & \multicolumn{2}{|c|}{$4 / 10$} & \multicolumn{2}{|c|}{$7 / 10$} \\
\hline Parenteral nutrition $(n)$ & \multicolumn{2}{|c|}{$3 / 10$} & \multicolumn{2}{|c|}{$0 / 10$} \\
\hline Enteral feeding $(n)$ & \multicolumn{2}{|c|}{$9 / 10$} & \multicolumn{2}{|c|}{$10 / 10$} \\
\hline \multicolumn{5}{|l|}{ Nutrition intake } \\
\hline Energy (kcal/kg) & 70 & $10-122$ & 82 & $5-129$ \\
\hline Carbohydrate $(\mathrm{g} / \mathrm{kg})$ & 9 & $2-14$ & 12 & $0.3-13$ \\
\hline Protein $(\mathrm{g} / \mathrm{kg})$ & $1 \cdot 7$ & $0.7-2.1$ & 0.5 & $0-3.9$ \\
\hline Fat $(\mathrm{g} / \mathrm{kg})$ & 3.8 & $0.4-10.2$ & 3.2 & $0.2-7.1$ \\
\hline Arginine (mg/kg) & 82 & $27-175$ & 119 & $14-145$ \\
\hline
\end{tabular}


Table 2. Plasma concentration of arginine, asymmetric dimethylarginine (ADMA), symmetric dimethylarginine (SDMA) and the arginine:ADMA ratio in premature infants with necrotizing enterocolitis (NEC) and without NEC (control)

(Median values and ranges)

\begin{tabular}{|c|c|c|c|c|c|}
\hline & \multicolumn{2}{|c|}{ NEC $(n 10)$} & \multicolumn{2}{|c|}{ Control (n 10) } & \multirow[b]{2}{*}{$P$ value } \\
\hline & Median & Range & Median & Range & \\
\hline Arginine $(\mu \mathrm{mol} / \mathrm{l})$ & 21.4 & $11 \cdot 5-59 \cdot 3$ & 55.9 & $27 \cdot 1-122 \cdot 3$ & 0.001 \\
\hline ADMA $(\mu \mathrm{mol} / \mathrm{l})$ & 0.59 & $0.33-1.02$ & 0.85 & $0.58-1.18$ & 0.009 \\
\hline SDMA $(\mu \mathrm{mol} / \mathrm{l})$ & 1.07 & $0.78-2.88$ & $1 \cdot 39$ & $0.74-2.05$ & NS \\
\hline Arginine:ADMA & $36 \cdot 6$ & $15 \cdot 6-94 \cdot 4$ & $72 \cdot 3$ & $35.9-103 \cdot 4$ & 0.023 \\
\hline
\end{tabular}

L-NMMA enhanced intestinal damage after administration of endotoxin. However, this damage could be reversed by administration of arginine (Hutcheson et al. 1990). The mucosal permeability of the feline ileum increased after infusion with the NO synthesis inhibitor L-NAME, while infusion with the NO donor sodium nitroprusside or arginine reversed this effect (Kubes, 1992). These animal studies indicate that a low plasma concentration of arginine, and inhibition of the arginine-NO pathway, could contribute to decreased synthesis of $\mathrm{NO}$, thereby resulting in decreased gastrointestinal blood flow and impairment of the intestinal mucosal barrier.

Interestingly, in contrast to our hypothesis, a significantly lower ADMA concentration was found in premature infants with NEC. Because ADMA is an endogenous inhibitor of all isoforms of NOS, a decrease of ADMA in premature infants with NEC could be associated with attempted regulatory changes aimed at a preservation of NO production in the presence of low arginine concentrations.

Although we cannot designate a single mediator for the observed effect, theoretically an increased metabolic turnover of ADMA may be responsible for the lower levels in NEC patients. ADMA is eliminated from the body by renal excretion as well as being metabolized by the enzyme dimethylarginine dimethylaminohydrolase (DDAH) which is widely distributed, in particular in the pancreas, liver and kidney (Kimoto et al. 1993; Kimoto et al. 1995). Induction of this enzyme or an increased renal excretion in NEC patients could be responsible for the decreased ADMA concentration (Yudkoff et al. 1984; Kimoto et al. 1995). Nijveldt and coworkers (Nijveldt et al. 2003b; Nijveldt et al. 2004) showed a reduced systemic ADMA concentration in rats during endotoxemia, indicating increased DDAH activity. Also, acute Escherichia coli endotoxaemia in human subjects decreased the plasma arginine:ADMA ratio (Mittermayer et al. 2004).
Since arginine and other cationic amino acids such as ornithine and lysine and also ADMA and SDMA are transported into endothelial cells by CAT, changes in the expression of CAT mRNA by endotoxemia may influence ADMA transport (Closs et al. 1997). Hattori et al. (1999), showed that expression of CAT 1 and 2 mRNA in the lung, heart, and kidney was increased by injection of lipopolysaccharide-interferon $\gamma$ in rats whereas CAT 2A mRNA was abundantly expressed in the liver independent of lipopolysaccharide-interferon $\gamma$ treatment. The abundant expression of CAT $2 \mathrm{mRNA}$ in the liver indicates a potentially high uptake of dimethylarginines in this organ. The important function of endotoxemia inducing $\mathrm{DDAH}$, as well as the expression of the CAT, provides a possible explanation for lower ADMA concentration in NEC patients.

Although the pathophysiology of NEC is multifactorial, there is some evidence suggesting that the development of NEC is associated with intestinal gram-negative bacterial growth (Neu, 1996; Neu, 2005; Westerbeek et al. 2006). Gram-negative bacteria produce the endotoxin lipopolysaccharide, inducing endotoxemia and the endogenous production of platelet activating factor and TNF (Feuerstein \& Hallenbeck, 1987; Beutler \& Cerami, 1988; Bone, 1991; Zanetti et al. 1992). The combination of lipopolysaccharide, platelet activating factor and TNF increases intestinal epithelial permeability and often causes necrosis which could contribute to the development of NEC (Hutcheson et al. 1990; Hsueh et al. 2003). Therefore, endotoxemia due to bacterial growth and release of lipopolysaccharide in premature infants can not only contribute to the development of NEC but can also induce DDAH and the expression of CAT, thereby increasing elimination of ADMA.

The lower concentration of ADMA could be a compensatory mechanism to counteract the disadvantageous effect of low arginine concentrations in NEC patients. However, since

Table 3. Plasma concentrations of arginine, asymmetric dimethylarginine (ADMA), symmetric dimethylarginine (SDMA) and the arginine:ADMA ratio in premature infants with necrotizing enterocolitis (NEC; non-survivors and survivors)

(Median values and ranges)

\begin{tabular}{lcccccc}
\hline & \multicolumn{2}{c}{ Non-survivors $(n 4)$} & & \multicolumn{2}{c}{ Survivors $(n 6)$} & \\
\cline { 2 - 3 } & Median & Range & & Median & Range & \\
\hline Arginine $(\mu \mathrm{mol} / \mathrm{l})$ & 14.7 & $11.5-16.4$ & & 33.8 & $17.7-59.3$ & 0.01 \\
ADMA $(\mu \mathrm{mol} / \mathrm{l})$ & 0.46 & $0.33-1.02$ & & 0.63 & $0.52-0.84$ & $\mathrm{NS}$ \\
SDMA $(\mu \mathrm{mol} / /)$ & 0.96 & $0.78-2.88$ & & 1.14 & $0.91-1.28$ & $\mathrm{NS}$ \\
Arginine:ADMA & 32.0 & $15.6-35.7$ & & 47.5 & $34.2-94.4$ & 0.038 \\
\hline
\end{tabular}


Table 4. Plasma concentrations of arginine, asymmetric dimethylarginine (ADMA), symmetric dimethylarginine (SDMA) and the arginine:ADMA ratio in surviving premature infants with necrotizing enterocolitis (NEC) and without NEC (control)

(Median values and ranges)

\begin{tabular}{|c|c|c|c|c|c|}
\hline & \multicolumn{2}{|c|}{ Survivors ( $n 6$ ) } & \multicolumn{2}{|c|}{ Control $(n 10)$} & \multirow[b]{2}{*}{$P$ value } \\
\hline & Median & Range & Median & Range & \\
\hline Arginine $(\mu \mathrm{mol} / \mathrm{l})$ & 33.8 & $17 \cdot 7-59 \cdot 3$ & $55 \cdot 9$ & $27 \cdot 1-122 \cdot 3$ & 0.022 \\
\hline ADMA $(\mu \mathrm{mol} / \mathrm{l})$ & 0.63 & $0.52-0.84$ & 0.85 & $0.58-1.18$ & 0.031 \\
\hline SDMA $(\mu \mathrm{mol} / \mathrm{l})$ & $1 \cdot 14$ & $0.91-1.28$ & 1.39 & $0.74-2.05$ & 0.022 \\
\hline Arginine:ADMA & 47.5 & $34.2-94.4$ & $72 \cdot 3$ & $35 \cdot 9-103 \cdot 4$ & NS \\
\hline
\end{tabular}

the decrease of arginine is larger than the decrease of ADMA, the arginine:ADMA ratio is lower in NEC patients, suggesting a reduced capacity for NO synthesis.

In this study we did not measure the oxidation products nitrate and nitrite as an indirect determination of endogenous formation of NO. Because the nitrate and nitrite concentration is influenced by endogenous NO synthesis, dietary intake and also by the excretion in urine, faeces and expired air, nitrate and nitrite concentration cannot be used as a truly meaningful indicator of $\mathrm{NO}$ production in premature infants (Baylis \& Vallance, 1998).

In conclusion, the present study confirms the existence of low arginine concentrations in premature infants with NEC. In contrast to our hypothesis, the ADMA concentration is lower in these infants. In spite of the low ADMA concentration, the arginine:ADMA ratio is lower in NEC patients than in controls. Furthermore, a low arginine plasma concentration and a low arginine:ADMA ratio are associated with an increased risk of mortality in NEC patients. These findings suggest that despite the increased need for arginine, premature infants with NEC have lower availability of this substrate to synthesize NO, which may be involved in the pathophysiology of NEC. The results of our study suggest the association between arginine, ADMA and NEC. However it remains to be determined whether low levels of arginine contribute to the pathophysiology of NEC, or if low arginine levels are a consequence of NEC. As suggested in the few other studies to date, we agree that the supplementation of arginine may prevent the development of NEC in premature infants. Due to the low incidence of NEC (on average 6 out of 300 patients per year), and a high local nosocomial infection rate in the VU Medical Center (van der Zwet et al. 2005) a multicentre randomized controlled study of arginine supplementation in premature infants is needed to determine whether arginine supplementation reduces the development of NEC.

\section{Acknowledgements}

The authors thank Ms Sigrid de Jong for measurement of the amino acids. M. C. Richir and M. P. C. Siroen contributed equally to this work.

\section{References}

Alican I \& Kubes P (1996) A critical role for nitric oxide in intestinal barrier function and dysfunction. Am J Physiol 270, G225-G237. Amin HJ, Zamora SA, McMillan DD, Fick GH, Butzner JD, Parsons HG \& Scott RB (2002) Arginine supplementation prevents necrotizing enterocolitis in the premature infant. $J$ Pediatr 140, $425-431$.

Baylis C \& Vallance P (1998) Measurement of nitrite and nitrate levels in plasma and urine - what does this measure tell us about the activity of the endogenous nitric oxide system? Curr Opin Nephrol Hypertens 7, 59-62.

Becker RM, Wu G, Galanko JA, Chen W, Maynor AR, Bose CL \& Rhoads JM (2000) Reduced serum amino acid concentrations in infants with necrotizing enterocolitis. J Pediatr 137, 785-793.

Bell MJ, Ternberg JL, Feigin RD, Keating JP, Marshall R, Barton L \& Brotherton T (1978) Neonatal necrotizing enterocolitis. Therapeutic decisions based upon clinical staging. Ann Surg 187, 1-7.

Beutler B \& Cerami A (1988) Tumor necrosis, cachexia, shock, and inflammation: a common mediator. Annu Rev Biochem 57, 505-518.

Bode-Boger SM, Boger RH, Kienke S, Junker W \& Frolich JC (1996) Elevated L-arginine/dimethylarginine ratio contributes to enhanced systemic NO production by dietary L-arginine in hypercholesterolemic rabbits. Biochem Biophys Res Commun 219, 598-603.

Boger RH, Bode-Boger SM, Thiele W, Creutzig A, Alexander K \& Frolich JC (1998) Restoring vascular nitric oxide formation by L-arginine improves the symptoms of intermittent claudication in patients with peripheral arterial occlusive disease. J Am Coll Cardiol 32, 1336-1344.

Bone RC (1991) The pathogenesis of sepsis. Ann Intern Med 115, 457-469.

Closs EI, Basha FZ, Habermeier A \& Forstermann U (1997) Interference of L-arginine analogues with L-arginine transport mediated by the y + carrier hCAT-2B. Nitric Oxide 1, 65-73.

Di Lorenzo M, Bass J \& Krantis A (1995) Use of L-arginine in the treatment of experimental necrotizing enterocolitis. Pediatr Surg 30, 235-240.

Dupont C (2003) Protein requirements during the first year of life. Am J Clin Nutr 77, 1544S-1549S.

Fan WQ, Smolich JJ, Wild J, Yu VY \& Walker AM (1996) Nitric oxide modulates regional blood flow differences in the fetal gastrointestinal tract. Am J Physiol 271, G598-G604.

Feuerstein G \& Hallenbeck JM (1987) Prostaglandins, leukotrienes, and platelet-activating factor in shock. Annu Rev Pharmacol Toxicol 27, 301-313.

Hattori Y, Kasai K \& Gross SS (1999) Cationic amino acid transporter gene expression in cultured vascular smooth muscle cells and in rats. Am J Physiol 276, H2020-H2028.

Hsueh W, Caplan MS, Qu XW, Tan XD, De Plaen IG \& GonzalezCrussi F (2003) Neonatal necrotizing enterocolitis: clinical considerations and pathogenetic concepts. Pediatr Dev Pathol 6, $6-23$.

Hutcheson IR, Whittle BJ \& Boughton-Smith NK (1990) Role of nitric oxide in maintaining vascular integrity in endotoxin-induced acute intestinal damage in the rat. Br J Pharmacol 101, 815-820.

Kimoto M, Tsuji H, Ogawa T \& Sasaoka K (1993) Detection of NG,NG-dimethylarginine dimethylaminohydrolase in the nitric 
oxide-generating systems of rats using monoclonal antibody. Arch Biochem Biophys 300, 657-662.

Kimoto M, Whitley GS, Tsuji H \& Ogawa T (1995) Detection of NG,NG-dimethylarginine dimethylaminohydrolase in human tissues using a monoclonal antibody. J Biochem (Tokyo) 117, 237-238.

Kliegman RM, Walker WA \& Yolken RH (1993) Necrotizing enterocolitis: research agenda for a disease of unknown etiology and pathogenesis. Pediatr Res 34, 701-708.

Kubes P (1992) Nitric oxide modulates epithelial permeability in the feline small intestine. Am J Physiol 262, G1138-G1142.

Mittermayer F, Namiranian K, Pleiner J, Schaller G \& Wolzt M (2004) Acute Escherichia coli endotoxaemia decreases the plasma L-arginine/asymmetrical dimethylarginine ratio in humans. Clin Sci (Lond) 106, 577-581.

Miyazaki H, Matsuoka H, Cooke JP, Usui M, Ueda S, Okuda S \& Imaizumi T (1999) Endogenous nitric oxide synthase inhibitor: a novel marker of atherosclerosis. Circulation 99, 1141-1146.

Nanayakkara PW, Teerlink T, Stehouwer CD, Allajar D, Spijkerman A, Schalkwijk C, Ter Wee PM \& van Guldener C (2005) Plasma asymmetric dimethylarginine (ADMA) concentration is independently associated with carotid intima-media thickness and plasma soluble vascular cell adhesion molecule-1 (sVCAM-1) concentration in patients with mild-to-moderate renal failure. Kidney Int 68, 2230-2236.

Neu J (1996) Necrotizing enterocolitis: the search for a unifying pathogenic theory leading to prevention. Pediatr Clin North Am 43, 409-432.

Neu J (2005) Neonatal necrotizing enterocolitis: an update. Acta Paediatr Suppl 94, 100-105.

Nijveldt RJ, Prins HA, Siroen MP, Rauwerda JA, Teerlink T \& van Leeuwen PA (2000) Low arginine plasma levels in patients after thoracoabdominal aortic surgery. Eur J Clin Nutr 54, 615-617.

Nijveldt RJ, Siroen MP, Teerlink T, van Lambalgen AA, Rauwerda JA \& van Leeuwen PA (2004) Gut and liver handling of asymmetric (ADMA) and symmetric (SDMA) dimethylarginine in the rat under basal conditions and during endotoxemia. Liver Int 24, 510-518.

Nijveldt RJ, Teerlink T, van der Hoven B, Siroen MPC, Kuik DJ, Rauwerda JA \& van Leeuwen PAM (2003a) Asymmetrical dimethylarginine (ADMA) in critically ill patients: high plasma ADMA concentration is an independent risk factor of ICU mortality. Clin Nutr 22, 23-30.

Nijveldt RJ, Teerlink T, van Guldener C, Prins HA, van Lambalgen AA, Stehouwer CD, Rauwerda JA \& van Leeuwen PA (2003b) Handling of asymmetrical dimethylarginine (ADMA) and symmetrical dimethylarginine (SDMA) by the rat kidney under basal conditions and during endotoxemia. Nephrol Dial Transplant 18, 2542-2550.

Schulze F, Lenzen H, Hanefeld C, et al. (2006) Asymmetric dimethylarginine is an independent risk factor for coronary heart disease: results from the multicenter Coronary Artery Risk Determination investigating the Influence of ADMA Concentration (CARDIAC) study. Am Heart J 152, 493-498.

Siroen MP, Teerlink T, Nijveldt RJ, Prins HA, Richir MC \& van Leeuwen PA (2006) The clinical significance of asymmetric dimethylarginine. Annu Rev Nutr 26, 203-228.

Stark ME \& Szurszewski JH (1992) Role of nitric oxide in gastrointestinal and hepatic function and disease. Gastroenterology 103, $1928-1949$.

Teerlink T, Nijveldt RJ, De Jong S \& van Leeuwen PA (2002) Determination of arginine, asymmetric dimethylarginine, and symmetric dimethylarginine in human plasma and other biological samples by high-performance liquid chromatography. Analytical Biochemistry 303, 131-137.

Vallance P \& Leiper J (2004) Cardiovascular biology of the asymmetric dimethylarginine:dimethylarginine dimethylaminohydrolase pathway. Arterioscler Thromb Vasc Biol 24, 1023-1030.

van der Zwet WC, Kaiser AM, van Elburg RM, Berkhof J, Fetter WP, Parlevliet GA \& Vandenbroucke-Grauls CM (2005) Nosocomial infections in a Dutch neonatal intensive care unit: surveillance study with definitions for infection specifically adapted for neonates. $J$ Hosp Infect 61, 300-311.

Westerbeek EA, van den BA, Lafeber HN, Knol J, Fetter WP \& van Elburg RM (2006) The intestinal bacterial colonisation in preterm infants: a review of the literature. Clin Nutr 25, $361-368$.

Wu G (1997) Synthesis of citrulline and arginine from proline in enterocytes of postnatal pigs. Am J Physiol 272, G1382-G1390.

Wu G, Jaeger LA, Bazer FW \& Rhoads JM (2004) Arginine deficiency in preterm infants: biochemical mechanisms and nutritional implications. J Nutr Biochem 15, 442-451.

Wu G \& Knabe DA (1995) Arginine synthesis in enterocytes of neonatal pigs. Am J Physiol 269, R621-R629.

Wu G \& Morris SMJ (1998) Arginine metabolism: nitric oxide and beyond. Biochem J 336, 1-17.

Yudkoff M, Nissim I, Pereira G \& Segal S (1984) Urinary excretion of dimethylarginines in premature infants. Biochem Med 32, $242-251$

Zamora SA, Amin HJ, McMillan DD, Kubes P, Fick GH, Butzner JD, Parsons HG \& Scott RB (1997) Plasma L-arginine concentrations in premature infants with necrotizing enterocolitis. $J$ Pediatr 131, $226-232$.

Zanetti G, Heumann D, Gerain J, Kohler J, Abbet P, Barras C, Lucas R, Glauser MP \& Baumgartner JD (1992) Cytokine production after intravenous or peritoneal gram-negative bacterial challenge in mice. Comparative protective efficacy of antibodies to tumor necrosis factor-alpha and to lipopolysaccharide. J Immunol 148, 1890-1897. 